Original scientific paper

\title{
VIBRATION REDUCTION ON OVERHEAD CONTACT RAILS: A SIMULATION-OPTIMIZATION APPROACH
}

\author{
Calvo Hernandez, A.; Sanz Bobi, J. de D.; Gomez Fernandez, J. \& Badolato Martin, A. \\ Universidad Politecnica de Madrid, Departamento Ingenieria Mecanica, C/ Jose Gutierrez Abascal 2, \\ 28006, Madrid, Spain \\ E-Mail: alvaro.calvo@upm.es, juandedios.sanz@upm.es, javier.gomezf@upm.es, \\ a.badolato@alumnos.upm.es
}

\begin{abstract}
The use of overhead contact rail systems in rail transport has been favoured by the need of having an electrification system that allows its installation where space is limited.

The displacement of a train pantograph running through a railway line causes an oscillating movement on the contact line. The overhead contact rail systems or rigid catenary, due to its characteristics, describes a movement with greater inertial forces than the equivalent flexible overhead contact lines. The main consequence is that at higher speeds larger variations in the contact force between pantograph and catenary wires occur, leading to contact loses and producing electrical arcs.

Results obtained in the study developed on the implementation of stiffness compensation systems are described on this paper. Simulations demonstrate that it is feasible to reduce the effects of the movement described by the overhead contact rail's beam. This opens a new investigation field focused on develop the most efficient configuration for alternative overhead contact rail solutions.

(Received in February 2021, accepted in April 2021. This paper was with the authors 2 weeks for 1 revision.)
\end{abstract}

Key Words: Pantograph-Catenary Interaction, Overhead Contact Rail, Vibration

\section{INTRODUCTION}

The installation of rigid or flexible catenary systems usually obeys two main criteria [1]. On one hand, an electrical criterion is imposed, which implies that the installed system should be capable of supplying the electricity demand necessary for the movement of the trains. This aspect directly constrains the distribution of the main elements that make up the railway's electric subsystem, including substations, autotransformers, supply lines and protection systems.

It must be considered that the electrical criterion hardly affects the constructive aspect of the contact line system, referring for example to the distribution of supports and droppers in the case of overhead contact line systems. This means that it should be considered that this constructive aspect has to also satisfy a mechanical criterion, which is necessary to consider in order to guarantee a correct transmission of the electric current.

In this respect, the mechanical criterion can make a bigger differentiation between overhead contact lines and overhead contact rail systems. The installation of one system or another can be defined in a simple way, since the overhead contact rail system is usually installed in tunnel areas or in places where the free space available is reduced.

In addition, contact elements between the trains' pantograph and the catenary should be considered: pantograph strips, generally made of copper alloys or carbon, and the wire of the catenary system, usually made of alloyed copper. Both elements are in direct contact continuously, ensuring the transmission of electrical current from one to another by applying an ascending force on the structure of the pantograph.

The ascending force mentioned is set with the train stopped, but the displacement of the trains and the dynamic behaviour of the systems that are in contact (pantograph and catenary) makes that contact force variable in time [2]. 
As demonstrated in previous studies [3-8], the wear of the elements exposed to friction can be estimated as the sum of the effects derived from the pressure between components due to the contact force and the effects derived from the electrical current transmission at the contact point. The latter generally produces an increase in temperature due to the high value of electrical resistance that appears at the contact point. Although, there is no independence between the two phenomena, since an increase in the contact force will mean greater wear due to abrasion of the materials but will reduce the amount of wear due to the transmission of electrical current since the contact area increases. These statements can be summarized as follows:

$$
\text { Wear }\left[\mathrm{mm}^{2}\right]=\frac{k_{F}}{\left(i[A]+i_{0}[A]\right)^{\frac{1}{n}}} \cdot F_{c}[N] \cdot V[m / s]+\frac{k_{i}}{\sqrt{F_{c}[N]}} \cdot i[A]^{2}
$$

Being the first term related to mechanical wear and the second term to electric wear, but both depending on the contact force $\left(F_{c}\right)$ and on the current transmission $(i) . k_{f}$ and $k_{i}$ are wear resistance coefficients that depend on the materials of the components in contact.

Vibrations in overhead contact systems are the main cause of wear in contact elements [9] and degradation of infrastructures [10] hence avoiding those oscillations will lead to greater lifetimes and reduction in maintenance activities. This reduction in times and expenses is the main objective of the elements described in this paper, based on its installation in existing infrastructures without changing the existing elements.

Studies developed on this research are based on algorithms and software that is specifically designed to reproduce a real situation. In this particular case, personnel involved from UPM (Universidad Politecnica de Madrid) has developed their own Matlab based algorithm that reproduces the dynamical behaviour of the contact components for both, contact line and contact rail system.

The algorithm mentioned above combines the use of FEM (Finite Elements Method), to define the infrastructure, with multibody theory, used for the definition of the pantograph. The combination of both methods into a single matrix structure allows the resolution of the stepped time advance, basing it on the Hilbert, Hughes \& Taylor (HHT) method [11-15].

\section{KEY ASPECTS OF INFRASTRUCTURE USAGE}

Present paper is focused on overhead contact rail systems. It can be observed that globally these kind of infrastructure has a speed limit depending on the support and span configurations. Larger distances between supports lead to vibrations with larger displacements of the overhead contact rail which can make the pantograph bounce against the contact rail.

Running speed limit can be set between $100 \mathrm{~km} / \mathrm{h}$ and $140 \mathrm{~km} / \mathrm{h}$ for classic contact rail systems with spans of 10 meters length. Above said limits, the catenary beam undergoes large variations in height in zones located between two consecutive supports. Considering the weight and the inertial forces derived from the beam movement, a significant variation of the contact force is produced.

Applying methods that maintain the extreme values of the contact force as close as possible to the static force value stablished for the pantograph will allow to not increase the adverse effects related to peak values. An excessive reduction in the contact force can serve as an example because it may lead to total contact losses, which generally result in the formation of electric arcs.

An electric arc would be the limit situation for the transmission of current, so it also produces high temperatures at the point where the arc occurs and, in most cases, material detachments from both the pantograph strips and the catenary system.

So far, the solutions proposed for the mitigation of the described problems generally involve the variation of the geometry of the catenary beam core [6], reducing its weight, the inertias 
derived from its displacement and the variation of the contact force between the copper components of both the pantograph and the catenary. Other solutions involve the installation of external dumpers on the top part of the catenary beam.

\section{PROPOSAL ON THE MANAGEMENT OF THE SYSTEM STIFFNESS}

Mechanism described below means by its characteristics as by its method of installation and operation, a variant not in up to now and that can be described as an intermediate solution between the systems of overhead contact rail and overhead contact lines. It should also be noted that the results presented are part of the technical feasibility studies carried out prior to the manufacturing, testing and commercialization process.

With the installation of the stiffness compensators it is intended to face two objectives of high interest within the railway systems.

On one hand, it is intended to improve operating conditions or in places where the wear of the friction elements is excessively high. Proposed system will allow to adapt the stiffness of the mechanical system that faces a pantograph in its displacement along a line, reducing the peaks of contact force between elements. Peak forces reduction may lead to better working condition and less wear in contact elements, allowing to reduce the maintenance activities related to parts replacement. Furthermore, supervision times may be rescheduled allowing to increase the working lifetime of the different elements.

On the other hand, as it is a system that can be considered as an interpolation between the catenary technologies currently available, it is intended to demonstrate that the installation of stiffness compensators would allow to increase the speed of the lines in places where the overhead contact rail currently imposes a limit around $100-140 \mathrm{~km} / \mathrm{h}$ in configurations with spans between supports of 10 meters in length.
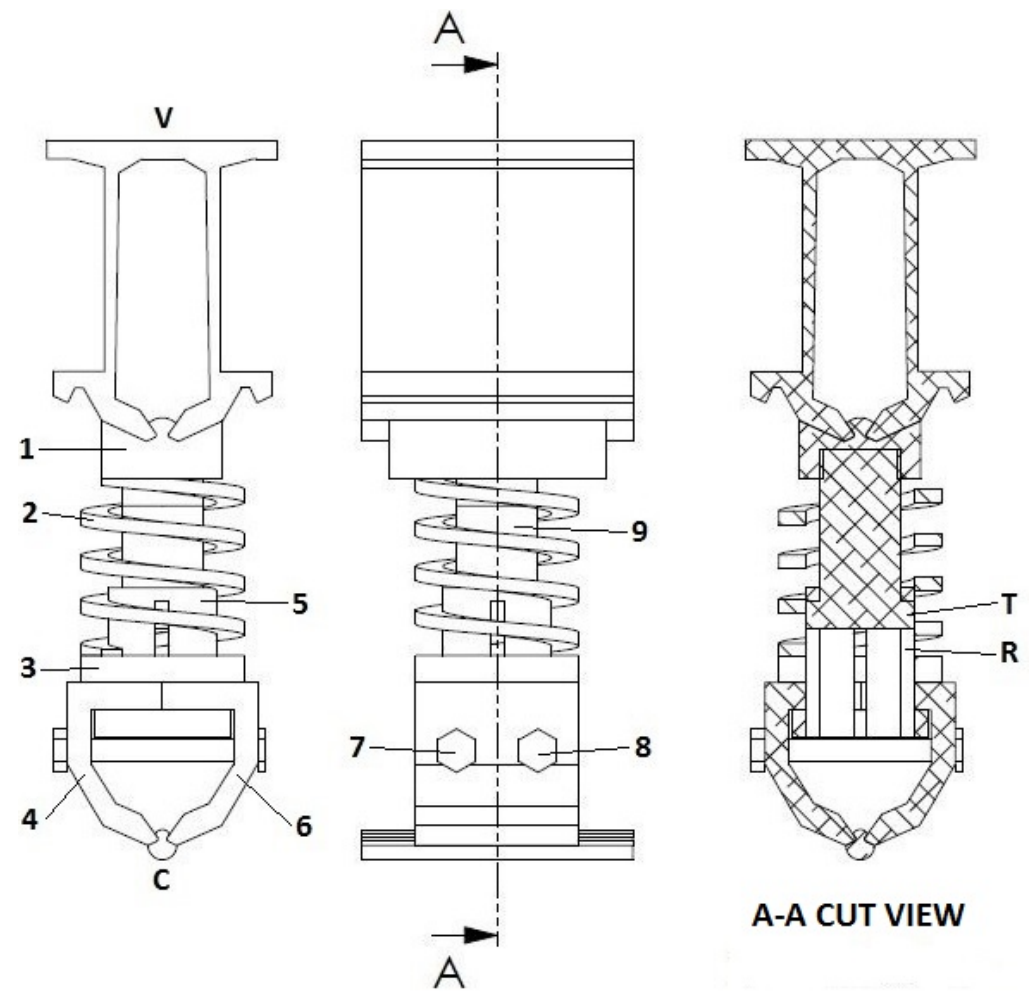

\section{A-A CUT VIEW}

Figure 1: Stiffness compensator front and side views including references.

Fig. 1 shows a schematic overview of the stiffness compensator proposed, where the following references have been set: 
- V: Overhead contact rail beam. Without special modifications. Placed on the figure to demonstrate the installation of the stiffness compensator.

- 1: Upper clamping element, inserted in the catenary beam just as currently fixation of the copper wire $\mathrm{C}$ is done.

- 2: Spring-type elastic element. The variation of the characteristics of this element will allow the variation of the stiffness of each installed compensator.

- 3: Elastomer element. Installed with the element 2, it allows the reduction of the vibrations generated when reaching the end of stroke in the extension movement of the stiffness compensator.

- 4 and 6: Clamps. Elements that allow the attachment of copper wire $\mathrm{C}$ to the compensator slide.

- 5: Slide. In its connection with the element 9 allows the movement of the lower parts with respect to the upper ones depending on the stiffness of the element 2.

- 7 and 8: Screw and nut union. It allows the clamping of the copper wire $\mathrm{C}$ and the blocking of the slide 5 with respect to clamps 4 and 6.

- 9: Guide for the slide.

The reference $\mathrm{T}$ are pins that allow the relative displacement of 9 and 5 when they are housed in the slots with reference $\mathrm{R}$.

The use of the elements R and T, as well as the geometry of the guide and the slider, allows to guarantee the vertical guidance of the deformations that take place between the copper wire and the aluminium beam, reducing the possibility of non-desired lateral displacements.

The Fig. 2 shows how the stiffness compensators should be installed between the aluminium beam and the copper wire. Further information and description about the device can be found at Spanish Patent Office [16].

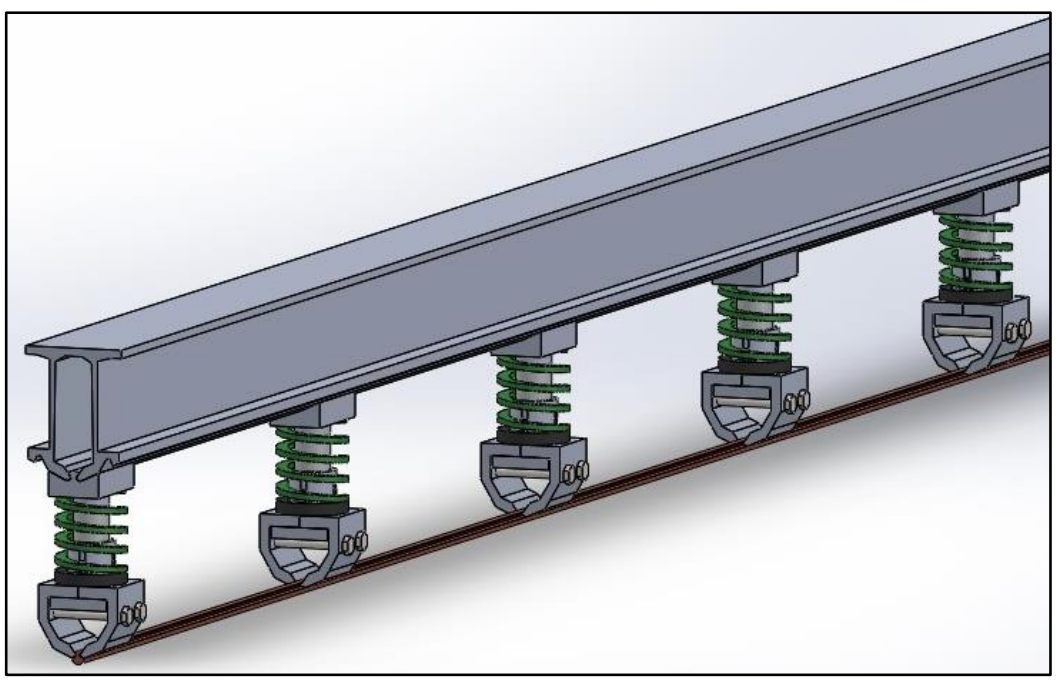

Figure 2: Stiffness compensators assembly.

\section{TECHNICAL FEASIBILITY OF THE PROPOSED SOLUTION}

In order to perform a first approximation of the behaviour of the stiffness compensators, a simulation procedure has been developed based on the combination of the theory of structures and the multibody simulation. For the resolution of the time problem, the algorithm of Hilber, Hughes \& Taylor (HHT) will be used. This method has proved its effectiveness during its development and subsequent testing period, closely related to project ALIS [17] (RTC-20153630-4) which has been considered as scientifically and technically favourable by Spanish Ministry of Science, Innovation and Universities on November $11^{\text {th }}, 2019$. 
HHT algorithm allows to calculate the displacement, speed and acceleration of the nodes of the overhead contact system structure. Equation stated is as follows:

$$
[M(t)] \cdot \ddot{x}(t+1)+[C(t)] \cdot \dot{x}(t+1)+[K(t)] \cdot x(t+1)=[F(t+1)]
$$

where $[\mathrm{M}]$ is the mass matrix, $[\mathrm{C}]$ is the dumping matrix and $[\mathrm{K}]$ is the stiffness matrix. Using this method, equations from overhead contact system and from pantograph can be built as a unique system and allows to set the conditions of the contact point through the force applied between contact surfaces and through the position and vertical speed of them.

Regarding the impacts that appear after a contact loss, simulation software detects this situation, reducing time step up to the moment of contact recovery. Once contact is recovered, time step is reduced once and again, and impact calculation process is repeated until impact forces for two consecutive time steps differ less than a $0.1 \%$. This procedure avoids false force values that may lead to unstable situations.

The validity of the results obtained using said simulation method has been contrasted with respect to the EN-50318:2018 standard [18] in the case of flexible catenary systems (overhead contact lines), and against actual records of installations in operation in the case of rigid catenary (overhead contact rail). As a side support, previous methodologies applied by UPM will be considered as a data source for adjusting the model and for data collection process [8]. In the specific case of the overhead contact line systems, results obtained are framed within the validity ranges of the simulation systems for dynamic interactions between pantograph and catenary collected in the EN-50318:2018 standard. This means that the method applied in the software developed is an appropriate tool for the simulation of these cases.

In the case of overhead contact rail systems, since there is no specific regulation at the time of writing this paper, an extrapolation of the standard EN-50318:2018 has been used as validation criterion, which validates the simulation systems by means of the comparison with respect to a measurement of a facility that is in a situation of regular use.

The criterion established for the comparison is that the simulation parameters such as average contact force or FM should not differ more than 2,5 N, and standard deviation of the contact force or $\sigma$ should not differ by more than $20 \%$, both of them with respect to the values obtained by direct measurement.

For this task, SBB (Schweizerische Bundesbahnen) has provided a real measurement of $240 \mathrm{~m}$ length section, with a span distance between supports of 10 meters.

This scenario has been reproduced in the simulation software, and comparison results are gathered in Table I. For the simulated value, a low pass filter of $400 \mathrm{~Hz}$ has been applied.

Table I: Comparison between characteristic values of contact force in real OCR and simulated systems.

\begin{tabular}{|l|c|c|c|}
\hline \multicolumn{1}{|c|}{ Variable } & Simulation result & Real value & Deviation \\
\hline Mean contact force & $80,87 \mathrm{~N}$ & $80,11 \mathrm{~N}$ & $1 \%$ \\
\hline Standard deviation & $10,34 \mathrm{~N}$ & $10,99 \mathrm{~N}$ & $6 \%$ \\
\hline Maximum contact force & $97,07 \mathrm{~N}$ & $111,87 \mathrm{~N}$ & $13 \%$ \\
\hline Minimum contact force & $59,81 \mathrm{~N}$ & $52,01 \mathrm{~N}$ & $15 \%$ \\
\hline
\end{tabular}

Values obtained by simulation using the algorithm described in overhead contact rail systems represent the same behaviour that the available measurement data when traveling speed is below $100 \mathrm{~km} / \mathrm{h}$ so it is assumed that the calculation system is also valid for these systems.

The method described will be finally extrapolated for its application on modified overhead contact rail systems by installing the stiffness compensators described above. 


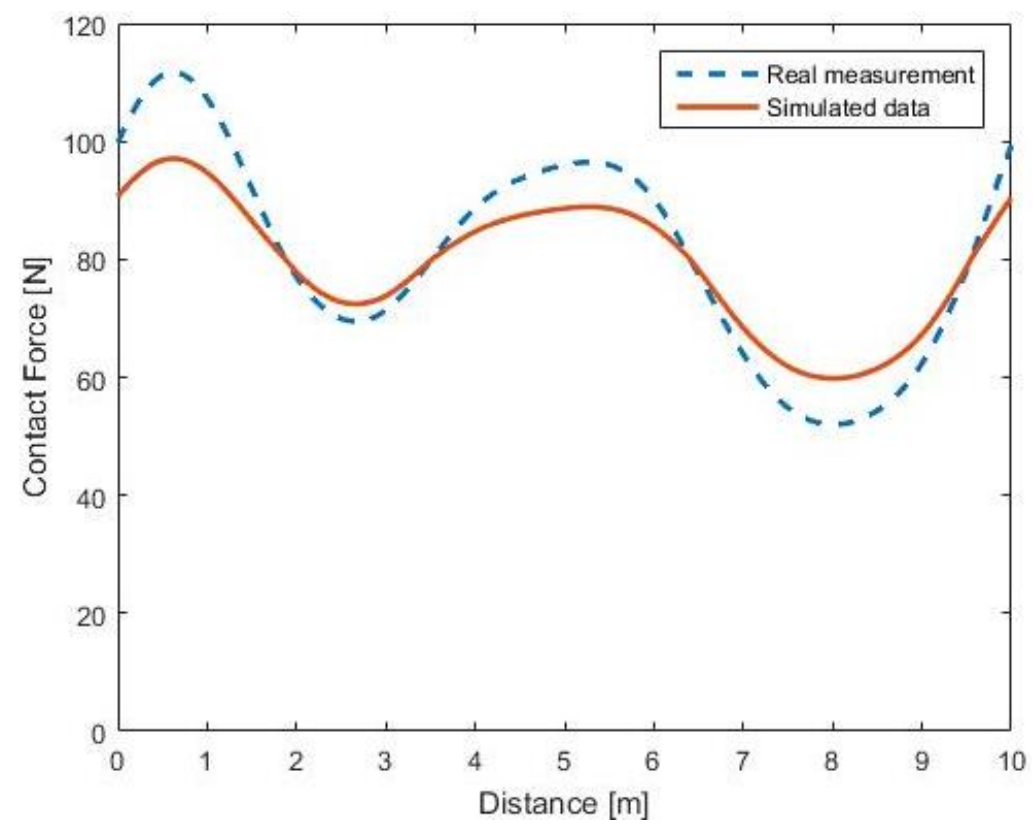

Figure 3: Comparison between real measurements and simulation data.

\section{METHODOLOGY}

It will be used as reference for this study an infrastructure with 24 consecutive spans of 10 meters in length and the results obtained will be considered only for the two central spans when making the relevant comparisons. This methodology has been chosen since it is similar to the one proposed in standard EN-50318:2018, the only reference available for the use of dynamic pantograph-catenary simulation software. Previous studies developed by UPM [8] also demonstrate that the chosen scenario is a representative situation of a regular contact rail installation.

The behaviour of a single pantograph will be considered in the study, a common situation when working with simple train compositions.

The adaptation of the finite element model to the overhead contact rail infrastructure has been made through the use of beam-type and bar-type elements. Bar-type elements will be considered for main supports and stiffness management devices, conditioned only by the dynamics of the movement in their vertical axis. In the case of copper cable and aluminium beam, beam-type elements become essential as stiffening effects appear due to shear stresses. The maximum length of the beam-type elements will be $0.01 \mathrm{~m}$. No more movement restrictions have been applied than those imposed by the structure described.

For the simulation of the behaviour of the pantograph, the reduced mass model that is used is the one included in the EN-50318:2018 standard.

Given that the use of overhead contact rail systems is usually considered as limited to maximum speeds of $100 \mathrm{~km} / \mathrm{h}$ when used in underground installations, this speed will be used as a reference for the first study, in search of replicating the mechanical characteristics of the interaction between pantograph and catenary in active overhead contact rail systems (OCR from now on) and the proposed modified overhead contact rail system (OCR-Mod from now on).

To achieve a similar response in the OCR-Mod systems, the installation of a stiffness compensator every half meter of catenary is proposed, so that in a 10-meter span there are 20 devices installed.

The possibility of varying the stiffness replacing the elastic element of the compensators will be the key aspect when adjusting the proposed system in different operating conditions. For tests developed, variation of stiffness goes from $30 \mathrm{kN} / \mathrm{m}$ to $50 \mathrm{kN} / \mathrm{m}$. 


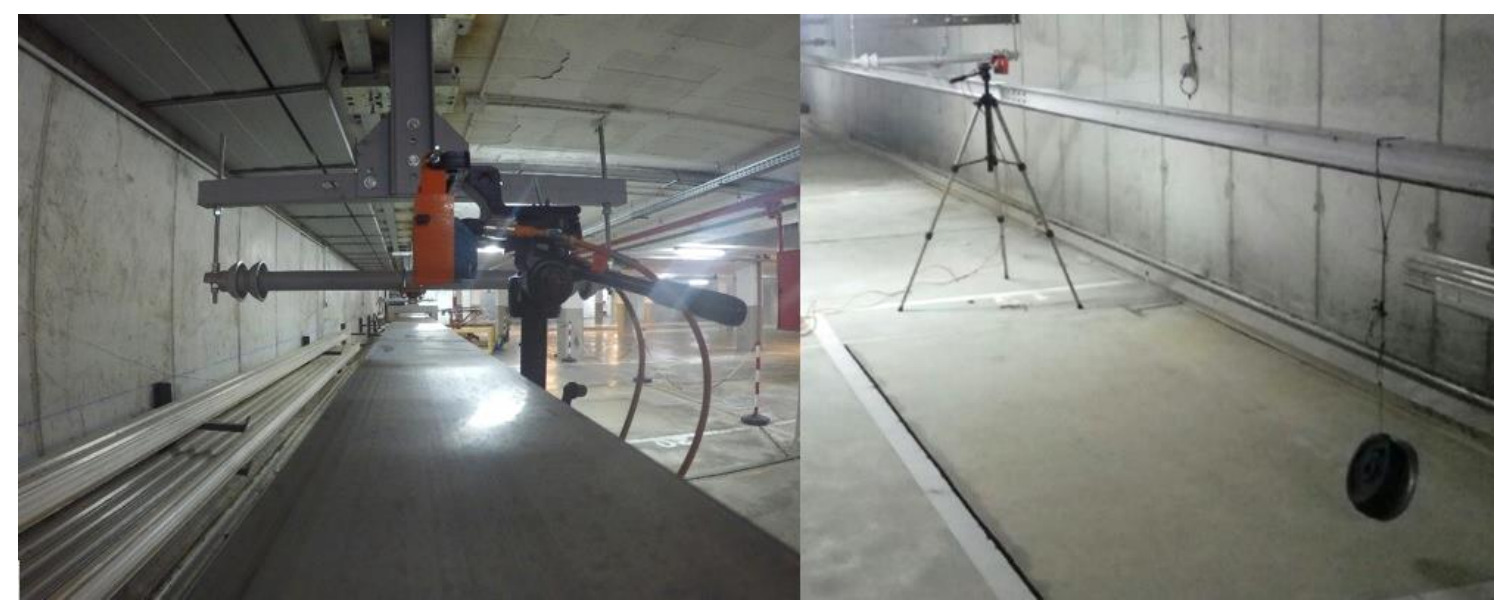

Figure 4: UPM tests at ADIF's facilities in Malaga.

Several tests have been carried out, as shown in Fig. 4, to verify which distribution offers the best dynamic results, being the most adequate the one that meets the following criteria:

- The devices located in the vicinity of the supports will be rigid enough to produce a minimum deformation of the device. This characteristic keeps the height of the contact wire, avoiding great variations on the whole structure.

- The elements located in the centre of the spans will be of less stiffness admitting greater deformation between the wire and the beam, but the stiffness should be enough so that the end of stroke is not reached in any situation.

- The elastomeric element will be identical in all devices.

The distribution of elements according to the previous pattern allows modifying the elasticity of the catenary at each point. The relative displacement between cable and beam allows a similar global vertical displacement of the cable at all points. The final effect can be compared with the difference that exists between overhead contact lines with simple hangers and with Y-hangers.

Table II compares the force required in each point to produce a vertical displacement of $1 \mathrm{~mm}$ in OCR and OCR-Mod systems referring the distance to the first support found while travelling. Data related to OCR systems were directly recorded by UPM in ADIF's testing bench placed in Malaga. Data related to OCR-Mod systems has been obtained by UPM modifying the elastic element of the different stiffness compensators.

Table II: Vertical stiffness in N/mm. Comparison between OCR and OCR-Mod systems.

\begin{tabular}{|l|c|c|c|c|c|c|c|c|c|}
\hline $\begin{array}{c}\text { Distance to } \\
\text { support }\end{array}$ & $\mathbf{1} \mathbf{~ m}$ & $\mathbf{2} \mathbf{~ m}$ & $\mathbf{3} \mathbf{m}$ & $\mathbf{4} \mathbf{~ m}$ & $\mathbf{5} \mathbf{~ m}$ & $\mathbf{6} \mathbf{~ m}$ & $\mathbf{7} \mathbf{~ m}$ & $\mathbf{8} \mathbf{~ m}$ & $\mathbf{9} \mathbf{~ m}$ \\
\hline OCR & 128 & 78,12 & 41,32 & 32,10 & 29,23 & 21,54 & 41,32 & 71,17 & 113,63 \\
\hline OCR-Mod & 150 & 135 & 125 & 120 & 120 & 120 & 125 & 135 & 150 \\
\hline
\end{tabular}

For OCR-Mod systems, elasticity of the overhead contact rail system becomes homogeneous producing almost the same displacement in each point.

Once the devices have been adjusted to achieve similar behaviours for OCR and OCR-Mod at $100 \mathrm{~km} / \mathrm{h}$, behaviour tests will be carried out at higher speeds. Specifically, $25 \mathrm{~km} / \mathrm{h}$ intervals from $100 \mathrm{~km} / \mathrm{h}$ to $250 \mathrm{~km} / \mathrm{h}$, minimum speed of the tests included in the EN-50318:2018 standard.

Desirable behaviours are those in which the applied average force is close to the static value of the contact force applied by the pantograph against the copper wire. In addition, it is positively valued that the standard deviation of the dynamic force is as small as possible.

Resulting from the latter, the minimum contact force should not be less than $50 \mathrm{~N}$ in any case in order to guarantee a correct collection of the electric current. 


\section{TESTING RESULTS}

The comparisons of the results obtained for the simulations carried out for both OCR systems and OCR-Mod systems are shown below. It should be considered that results correspond to the stiffness compensators configuration that has presented the best results up to date. It is considered appropriate not to include the tests that do not provide significant information.

Fig. 5 shows a comparison of the behaviour of the reaction force of contact between two consecutive supports. This figure allows to understand the significant variation of the dynamical behaviour of the contact rail systems when stiffness compensators are included in the infrastructure or not. This figure corresponds to the test with a traveling speed of $250 \mathrm{~km} / \mathrm{h}$.

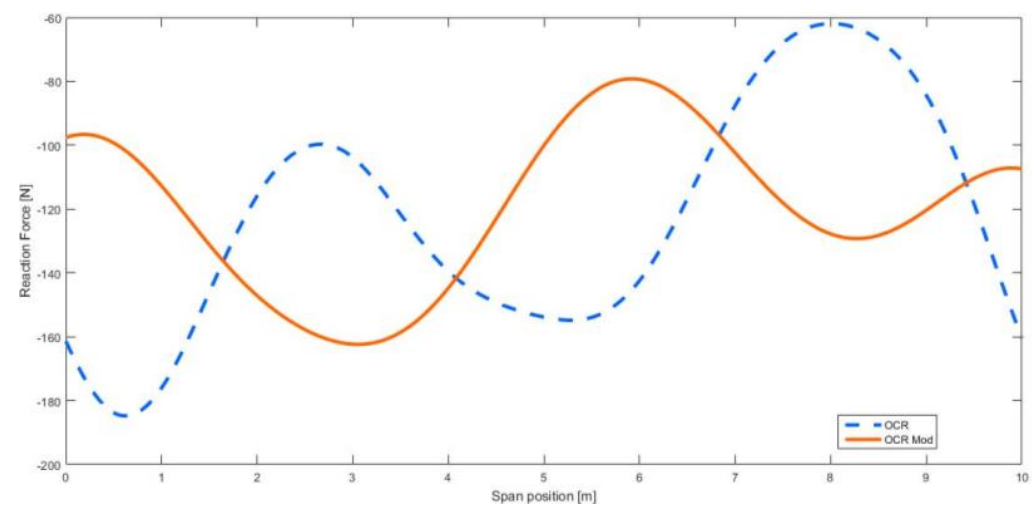

Figure 5: Contact force behaviour comparison in OCR and OCR-Mod systems.

First, the average force applied during the contact process is compared. The starting point is a static force of $120 \mathrm{~N}$, which, although it may be slightly elevated for a rigid catenary system, is the only standardized reference for a pantograph.

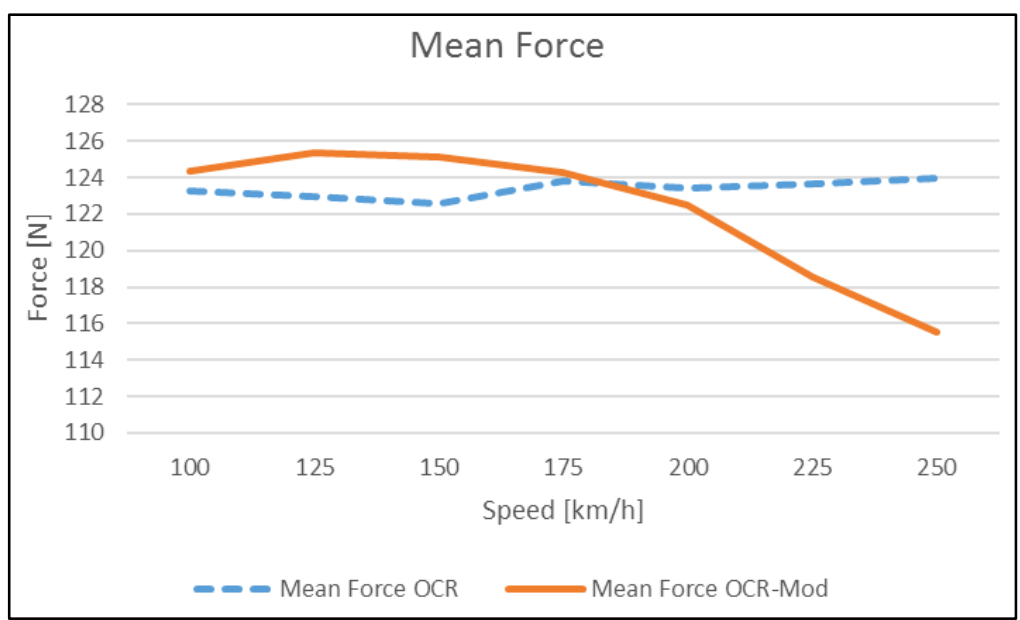

Figure 6: Comparison between mean contact force values in OCR and OCR-Mod systems.

As shown in Fig. 6, when using an OCR-type catenary, the dynamic averaged contact force hardly changes with respect to the static force of $120 \mathrm{~N}$ set for the test. It is important not to mix the concept of averaged force applied with the real contact force, since the real force is shown in the previous graph. The undulations shown are mainly due to the aerodynamic forces that appear on the pantograph plus the reaction forces that appear in the contact between components.

For the OCR-Mod type catenary, a reduction of the averaged contact force is observed as the speed increases. This reduction does not lead to risky limits for the transmission of electric current, but it can help to reduce the rate of wear related to the friction of the elements in contact. 


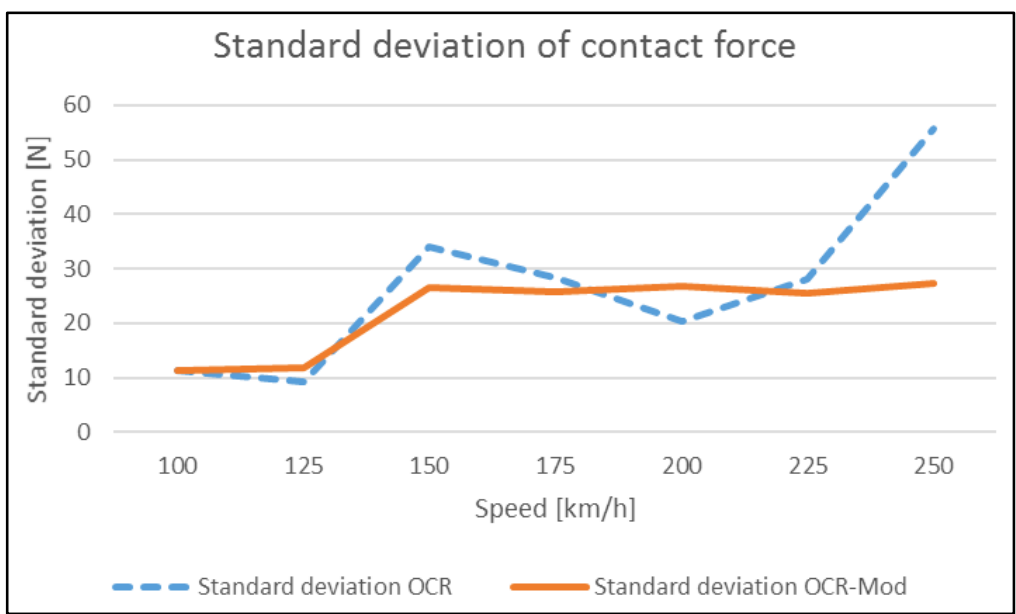

Figure 7: Comparison between contact force's standard deviation values in OCR and OCR-Mod systems.

Secondly, Fig. 7 shows the standard deviation of the contact force for different speeds. In this case the objective is that the maximum and minimum values of the dynamic contact force are as close as possible to the averaged contact force value, which will lead to lower values of the standard deviation.

It can be observed that the OCR systems show an erratic behaviour with the increase of speed, obtaining very high values of dispersion followed by very low values. This situation prevents to reach high speeds, since reaching a speed above the first point where the dispersion is high involves going through an area of instability in the contact that can lead to contact losses or reduction of the capacity of electrical current collection.

Implementation of an OCR-Mod system proves to be especially useful in this field, significantly reducing the randomness of the mentioned behaviour and stabilizing the value of the standard deviation around reduced values for any speed that is considered. It is also observed that, the higher the circulation speed is, the greater the stabilizing effect is observed on the system in which the stiffness compensators are installed.

As a consequence of the effects described for the average contact force and for the standard deviation of it, the behaviour corresponding to the registered maximum and minimum values can be observed in Figs. 8 and 9.

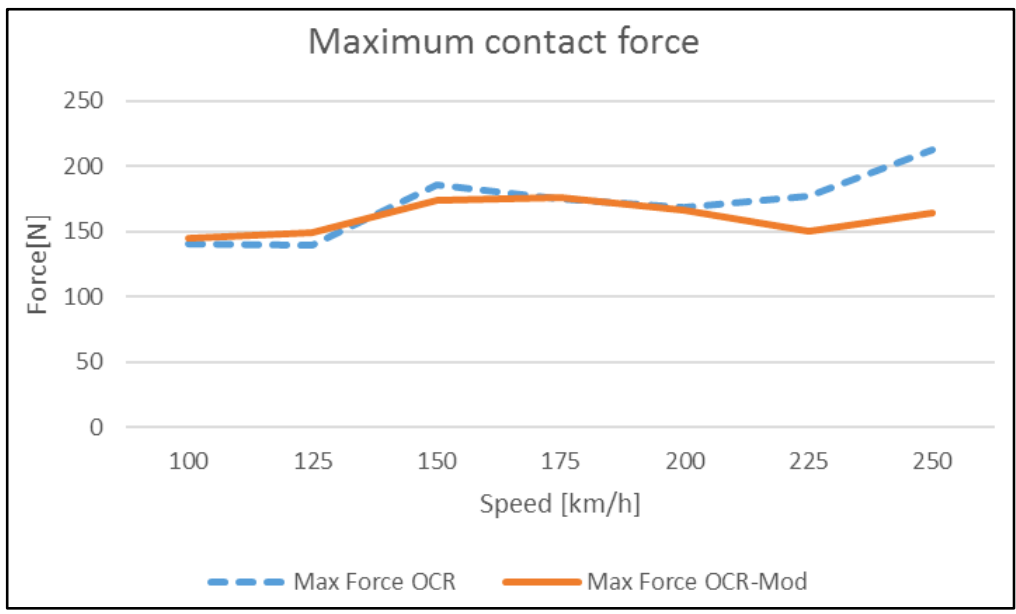

Figure 8: Comparison between maximum values of contact force in OCR and OCR-Mod systems. 


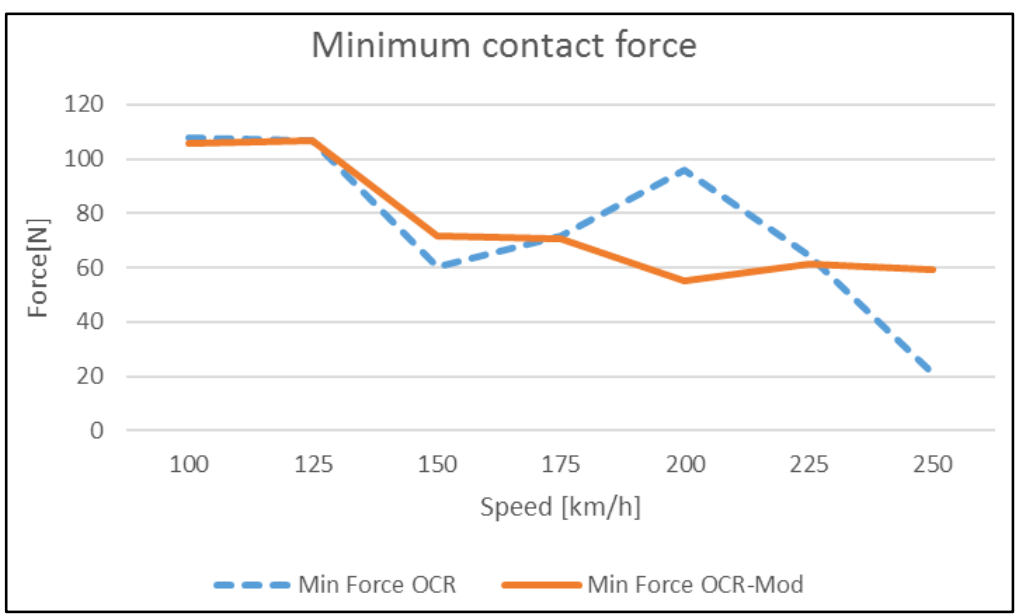

Figure 9: Comparison between minimum values of contact force in OCR and OCR-Mod systems.

In the case of the maximum value recorded, it can be seen that with the increase in speed the OCR-Mod system tends to have lower maximums than the OCR system, a condition that will reduce the wear of the elements subjected to friction. In the Fig. 7 it can be verified that the variation does not become especially significant until reaching speeds above $200 \mathrm{~km} / \mathrm{h}$.

Unlike what was observed in the case of the maximum force values, the behaviour of the minimum forces applied show a direct relation with speed. Greater variations can be seen from $125 \mathrm{~km} / \mathrm{h}$ onwards.

It can be seen that the effect of the installation of stiffness compensators produces an effect that shifts from a not predictable behaviour in the case of OCR systems to a behaviour that depends on speed on OCR-Mod systems.

It can also be observed that for the specific case of running at $250 \mathrm{~km} / \mathrm{h}$ the minimum force in the OCR-Mod systems maintains a force significantly higher than the obtained with a normal OCR system, being able to guarantee the correct collection of electric current between the catenary and the pantograph. In addition, the OCR-Mod system does not present forces below $50 \mathrm{~N}$ in the speed range under study.

The question may arise as to whether the simulation method affects the results obtained. As explained above, the method applied and the simulation criteria have multiple prior references, some of them specially focused on simulation for speeds over $140 \mathrm{~km} / \mathrm{h}$ [8] and on the design of alternative contact rail systems [6], both works developed by UPM.

The contact rail design proposed by UPM [6] previously allowed to reduce the vertical inertial forces of the contact rail beam, maintaining a unique profile along the entire line and was implemented on Line 1 of Metro de Madrid. The modification that is proposed in this paper allows, by varying the stiffness of the elastic element in each device, to create variable stiffness profiles between supports. This modification mainly reduces the displacement of the contact rail beam that occurs at each contact point, also reducing the variability of the contact force.

The behaviour described in the previous paragraph allows to conclude that there will be a specific configuration of stiffness compensators for each location, mainly depending on the running speed of trains. Authors are currently working on suitable configurations for the entire range of speeds existing in the Spanish railway.

\section{CONCLUSIONS}

Considering results obtained, the installation of stiffness compensators in overhead contact rail systems presents a feasible way to improve the dynamic response of the current collection system. 
This improvement is especially evident above $125 \mathrm{~km} / \mathrm{h}$, avoiding behaviours that a priori do not have a direct relationship with the running speed of trains and making these behaviours easily predictable.

Moreover, a fine adjust of the stiffness of the new components may allow to reduce the averaged contact force up to $9 \%$ at $250 \mathrm{~km} / \mathrm{h}$, and the standard deviation of the contact force up to $50 \%$ at $250 \mathrm{~km} / \mathrm{h}$. This implies lower maintenance costs due to the lower wear of the parts involved in the contact between pantograph and OCR systems.

These effects are of special interest in order to increase the running speed in railway lines that have overhead contact rail systems, maintaining the quality of the electric current collection without significantly increasing the wear levels of the contact elements.

\section{AKNOWLEDGEMENTS}

Special thanks to ADIF (Administrador de Infraestructuras Ferroviarias), and specially to Miguel Ríos Reyes for allowing the use of their testing bench of Overhead Contact Rail placed at Málaga's Railway Technology Center (CTF, Centro de Tecnologías Ferroviarias) and to SBB (Schweizerische Bundesbahnen), and specially to Uwe Schönherr, for allowing the use of data relative to dynamic forces and quality of current transmission in overhead contact rail installations already in use.

\section{REFERENCES}

[1] Kiessling, F.; Puschmann, R.; Schmieder, A.; Schneider, E. (2018). Contact Lines for Electric Railways, $3^{\text {rd }}$ edition, Publicis Publishing, Munich

[2] EN 50119:2010 (2010). Railway applications - Fixed installations - Electric traction overhead contact lines, CENELEC, Brussels

[3] Poetsch, G.; Evans, J.; Meisinger, R.; Kortüm, W.; Baldauf, W.; Veitl, A.; Wallaschek, J. (1997). Pantograph/catenary dynamics and control, Vehicle System Dynamics: International Journal of Vehicle Mechanics and Mobility, Vol. 28, No. 2-3, 159-195, doi:10.1080/00423119708969353

[4] Koyama, T.; Aboshi, M. (2013). Formation mechanism of undulating wear on overhead conductor rails due to dynamic characteristics of pantographs, Quarterly Report of RTRI, Vol. 54, No. 1, 1823, doi:10.2219/rtriqr.54.18

[5] Gonzalez, F. J.; Chover, J. A.; Suarez, B.; Vazquez, M. (2008). Dynamic analysis using finite elements to calculate the critical wear section of the contact wire in suburban railway overhead conductor rails, Proceedings of the Institution of Mechanical Engineers, Part F: Journal of Rail and Rapid Transit, Vol. 222, No. 2, 145-157, doi:10.1243/09544097JRRT144

[6] Vera, C.; Suarez, B.; Paulin, J.; Rodríguez, P. (2006). Simulation model for the study of overhead rail current collector systems dynamics, focused on the design of a new conductor rail, Vehicle System Dynamics: International Journal of Vehicle Mechanics and Mobility, Vol. 44, No. 8, $595-$ 614, doi:10.1080/00423110500165499

[7] Vera, C.; Paulin, J.; Suarez, B.; Rodríguez, P. (2005). Improved design of an overhead rail current conductor for application in underground lines, Proceedings of the $8^{\text {th }}$ International Conference on Railway Engineering: Maintenance \& Renewal of Permanent Way; Power \& Signaling; Structures \& Earthworks, London, 15 pages

[8] Chover, J. A.; Suarez, B.; Rodríguez, P. (2011). Simulation techniques for design of overhead conductor rail lines for speeds over $140 \mathrm{~km} / \mathrm{h}$, Proceedings of the $22^{\text {nd }}$ International Symposium on Dynamics of Vehicles on Roads and Tracks, Manchester, 6 pages

[9] Zang, J. W.; Wu, J. Q.; Chen, W. R.; Guan, J. F.; Zhong, Y.; Xu, K. J. (2019). Simulation method for dropper dynamic load considering horizontal vibration behaviour, International Journal of Simulation Modelling, Vol. 18, No. 4, 620-631, doi:10.2507/IJSIMM18(4)492

[10] Szurgott, P.; Bernacki, P. (2020). Modelling of steel-concrete bridges subjected to a moving highspeed train, International Journal of Simulation Modelling, Vol. 19, No. 1, 29-40, doi:10.2507/ $\underline{\text { IJSIMM19-1-499 }}$ 
[11] Bautista, A.; Montesinos, J.; Pintado, P. (2015). Dynamic interaction between pantograph and rigid overhead lines using a coupled FEM - multibody procedure, Mechanism and Machine Theory, Vol. 97, 100-111, doi:10.1016/j.mechmachtheory.2015.10.009

[12] Rauter, F. G.; Pombo, J.; Ambrosio, J.; Chalansonnet, J.; Bobillot, A.; Pereira, M. S. (2007). Contact model for the pantograph-catenary interaction, Journal of System Design and Dynamics, Vol. 1, No. 3, 447-457, doi:10.1299/jsdd.1.447

[13] Pombo, J.; Ambrosio, J. (2012). Influence of pantograph suspension characteristics on the contact quality with the catenary for high speed trains, Computers \& Structures, Vols. 110-111, 32-42, doi:10.1016/j.compstruc.2012.06.005

[14] Seo, J.-H.; Kim, S.-W.; Jung, I.-H.; Park, T.-W.; Mok, J.-Y.; Kim, Y.-G.; Chai, J.-B. (2006). Dynamic analysis of a pantograph-catenary system using absolute nodal coordinates, Vehicle System Dynamics: International Journal of Vehicle Mechanics and Mobility, Vol. 44, No. 8, 615630, doi:10.1080/00423110500373721

[15] Big-Alabo, A.; Ossia, C. V. (2019). Analysis of the coupled nonlinear vibration of a two-mass system, Journal of Applied and Computational Mechanics, Vol. 5, No. 5, 935-950, doi:10.22055/ jacm.2019.28296.1474

[16] Calvo, H. A.; Sanz, B. J. D. (2019). Compensador de rigidez para sistemas de catenaria rígida, Patent ES2683778B2, Spanish Patent Office, Madrid

[17] Universidad Politécnica de Madrid, Abengoa, Universidad de Málaga. ALIS: Modelado basado en Algoritmos Inteligentes para la Integración de la electrificación, seguridad y eficiencia energética en sistemas ferroviarios (RTC-2015-3630-4), from http://www.laenergiadelcambio.com/alisherramienta-simulacion-entorno-electrificacion-ferroviarial, accessed on 31-01-2021

[18] EN 50318:2018 (2018). Railway applications - Current collection systems - Validation of simulation of the dynamic interaction between pantograph and overhead contact line, CENELEC, Brussels 